\title{
«Wir brauchen einen liberaleren Umgang mit Patientendaten»
}

\section{Felicitas Witte}

Dr. med., Medizinjournalistin

Schweizer hätten zu viel Angst um ihre Daten, das habe Nachteile für die Forschung. Andererseits müsse man diese Angst ernst nehmen. Auf dem 16. "forumsante.ch»* diskutierten Experten aus Medizin und Politik über die Transparenz von Gesundheitsdaten. Fazit: Daten müssen geschützt werden, aber sie können die Therapie sicherer und besser machen.

Alle möglichen Daten über uns geben wir in Facebook \& Co. preis. Aber wenn es darum geht, dass der Hausarzt uns einen Arztbrief per E-Mail schickt, bekommen wir Panik, unsere Daten könnten missbraucht werden. «Wie viel Datenschutz ist ungesund?», fragte Jacques de Haller, ehemaliger Präsident der FMH, und lud zu einem «forumsante.ch" zu diesem Thema.

Ein Kongress zum Thema Datenschutz war schon lange notwendig. In der Bevölkerung grassiert eine Angst, die ansteckt und panisch macht, aber ist sie berechtigt? Es geht um die Transparenz von Gesundheitsdaten. Apps zeichnen unsere Joggingstrecken auf, messen unsere Herzfrequenz und wie viele Schritte wir pro Tag gehen. Kaum jemand fragt sich, wohin diese Daten gelangen und was Google, Facebook, Apple \& Co. damit machen. Doch soll man seine Blutprobe für Studien dauerhaft zur Verfügung stellen, regen sich viele auf. De Haller: «Wir haben in der Schweiz viel zu viel Angst um unsere Daten", sagte de Haller. «In den skandinavischen Ländern gibt es schon seit Jahrzehnten Register mit anonymisierten
Patientendaten, und die Kollegen können damit exzellente Forschung machen.» Andererseits müsse man natürlich die Angst ernst nehmen. Unreflektiert sollte man seine Daten nicht überall preisgeben.

\section{Hippokrates: eine Fiktion seit der Antike}

Vor 2500 Jahren habe Hippokrates die Grundlage für die Sicherheit von Patientendaten geschaffen, in Form des hippokratischen Eides, sagte de Haller. «Wir müssen überlegen, welche Aspekte noch aktuell und schützenswert und welche total überholt sind. Es gibt nämlich einige Vorteile, welche die Lockerung des Arztgeheimnisses mit sich bringen könnte.»

Der hippokratische Eid gelte heute immer noch als Qualitätskriterium, erzählte Hubert Steinke, Direktor des Instituts für Medizingeschichte an der Uni Bern. «Es gibt aber überhaupt keinen Hinweis, dass Hippokrates den Eid oder irgendeinen ähnlichen Text verfasst hat», sagte Steinke und räumte damit mit einer Annahme auf, die sich bei Medizinern und Nichtmedi-

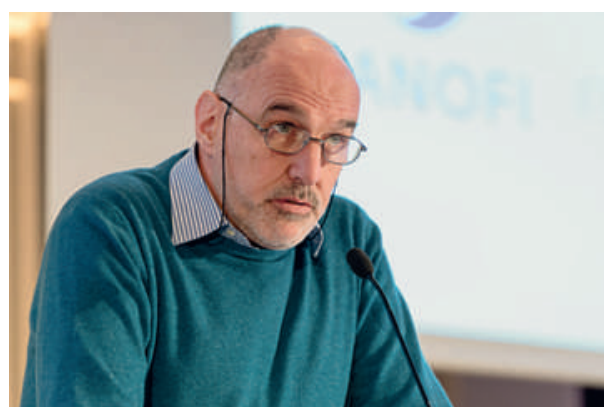

Jacques de Haller: «Beim Thema Transparenz von Patientendaten müssen wir uns aus der Zwangsjacke «Es muss alles partout geschützt werdens befreien.»

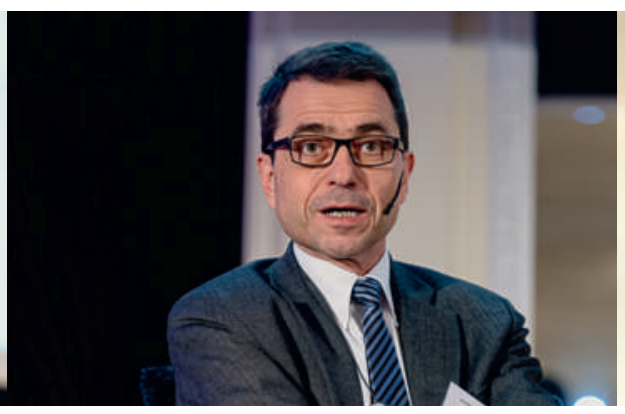

Oliver Peters: «Eine Versichertenkarte mit Chip macht zum Beispiel den Informationsaustausch zwischen Leistungserbringern zwar möglich, gewährleistet ihn aber nicht.»

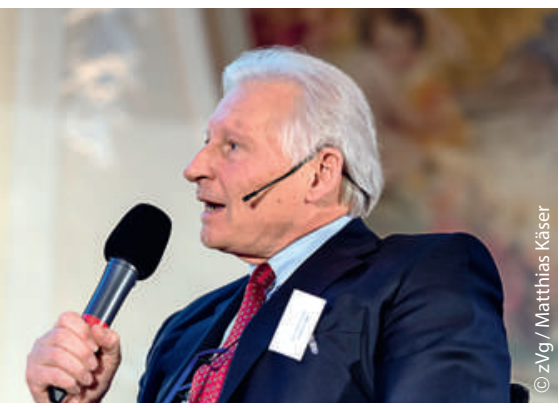

Urs Stoffel: «Datenschutz lässt sich nur durch Eigenverantwortung und eine gemeinsame Strategie umsetzen. Gesetze und Verbote nützen nichts.» 
zinern hartnäckig hält. «Hippokrates ist eine Fiktion seit der Antike. Er ist der Idealtypus des vollkommenen Arztes, der die ganze Breite der Medizin umfasst, Theorie und Praxis vereint und nach höchsten ethischen Prinzipien handelt.» Hippokrates - nur eine Fiktion? Dann müsste es doch leicht fallen, das Arztgeheimnis zu lockern, dachten wohl einige Zuhörer.

\section{Patienten von eHealth überzeugen}

Die elektronische Übermittlung und Speicherung von Patientendaten kann diesen durchaus nützen, sagte Oliver Peters, Vizedirektor des Bundesamtes für Gesundheit. «Die meisten sind zur Datenübermittlung und sehr viele auch zur dauerhaften Datenspeicherung bereit, wenn man ihnen erklärt, was das für einen Mehrwert für sie hat.» eHealth, also Behandlungsprozesse digital unterstützen, würde einerseits dem Patienten nützen, denn Behandlungen würden sicherer und besser werden. Zum Beispiel, weil die Medikation des Patienten stets dokumentiert ist und der Arzt automatisch vor allfälligen Wechselwirkungen gewarnt wird. Oder weil alle Massnahmen am Patienten digital dokumentiert werden, so dass keine Pflegekraft mehr die unleserliche Schrift eines Arztes dechiffrieren muss. Oder weil alle Informationen jederzeit abrufbar sind und der Patient keine unnötigen Doppeluntersuchungen über sich ergehen lassen muss. "Aber auch die Leistungserbringer würden deutlich profitieren", sagte Peters. Die für eine Therapie notwendigen Informationen wären ständig verfügbar und Behandlungen liessen sich besser nachvollziehen - ob das jedem Arzt gefallen würde, steht allerdings auf einem anderen Blatt. Das Spital profitiere, weil sich Klinikpfade besser steuern liessen, weil die Qualität der Diagnosen verbessert würde und weil medizinisches Personal besser ausgebildet werden könne. Der niedergelassene Arzt hätte weniger Aufwand mit Papierdossiers und Ablage, seine Dokumentation sei gesichert und er könne leichter mit Versicherungen oder Spitälern kommunizieren. "Im Zusammenhang mit eHealth wird viel versprochen, aber oft wird ein potentieller mit einem realen Nutzen verwechselt», gab Peters jedoch zu bedenken. So würden die meisten IT-Projekte auf der Basis eines potentiellen Nutzens verkauft und beschlossen. «Eine Versichertenkarte mit Chip macht zum Beispiel den Informationsaustausch zwischen Leistungserbringern zwar möglich, gewährleistet ihn aber nicht», sagte Peters. «Und eine Operationszentrum-Management-Software ermöglicht eine gute Planung von Operationen, garantiert sie aber nicht. Der potentielle Nutzen von IT-Instrumenten wird aber nur rea- lisiert wenn diese flächendeckend und systematisch eingesetzt werden.» Da grosse, arbeitsteilige Leistungserbringer wie Spitäler, Pflegeheime oder SpitexOrganisationen am ehesten direkt an der Einführung von eHealth-Lösungen interessiert seien, habe es Sinn, ausgehend von diesen Institutionen die Patientenprozesse digital zu unterstützen. Einzelne Kantone versuchen diesen Weg bereits in kantonalen eHealth-Strategien umzusetzen. Eine weitere Möglichkeit sei, den Einsatz von eHealth-Lösungen attraktiver zu gestalten beziehungsweise notwendig zu machen. So könnte als Vorgabe gelten, dass Einweiser diagnostische Informationen und die vorbestehende Medikation bei Eintritt oder Transfer in ein Spital in einem standardisierten und lesbaren Format übermitteln und dass der Spital-

Die meisten sind zur Datenübermittlung bereit, wenn man ihnen erklärt, welchen Mehrwert das für sie hat.

arzt den Austrittsbericht innerhalb von maximal 24 Stunden nach Entlassung sendet. "Um den Nutzen von eHealth-Systemen zu realisieren, genügt es nicht, die Patienten zu überzeugen", sagte Peters, "sondern es muss investiert werden: in die Organisation, in eine erste Akkumulation von Patientendaten und in die Anforderungen an Prozesse und Schnittstellen.» Nicht klar ist, wie Patienten wirklich zum Thema Datenfreigabe stehen. Dass einige dazu gar nicht überredet werden müssten, sondern die Weitergabe im Gegenteil fördern wollen, leuchtete nach dem Vortrag von Rainald von Gizycki ein. Gizycki ist Vertreter von Eurordis, dem Europäischen Verband von Patienten mit Seltenen Krankheiten. Ihm wurde 1972 die Diagnose Retinopathia pigmentosa mitgeteilt, eine unheilbare Augenkrankheit, die mit fortschreitendem Visusverlust einhergeht. "Der Patient möchte seine Daten weitergeben», sagte Gizycki. "Er muss sich unproblematisch eine Zweit- und Drittmeinung einholen können. Er muss die Daten in eigener Hand haben, um sie zum Beispiel seinem Arbeitgeber erklären zu können. Und damit er sie an Versicherungen, Versorgungsämter oder Rehaeinrichtungen senden kann.» Wie bei vielen seiner Leidensgenossen wurden bei ihm immer wieder Elektro-Retinogramme angefertigt, die eigentlich überflüssig gewesen wären, wenn die Ärzte die Daten gehabt hätten. «Patienten wollen keine Doppel-Untersuchungen. Bitte, liebe Ärzte, nutzt die Daten, die wir mitbringen.»

\section{NewIndex: ärzteeigener Datenpool}

Müsse man Daten schützen oder sich vor den Daten schützen, fragte provokant Urs Stoffel, Mitglied des 
Zentralvorstandes der FMH. 30 Minuten nach der Operation würden Patienten bereits Fotos auf Facebook posten, und das Selfie im Spital gehört für viele Jugendliche längst dazu. Durch unser Konsumverhalten bekommen Apotheken, Coop oder Migros einen detaillierten Überblick über unser Gesundheitsverhalten, Kreditkartenunternehmen nutzen die Kenntnisse über unsere Einkäufe und steigen ins Lebensversicherungsgeschäft ein. "Datenschutz lässt sich nur durch Eigenverantwortung und eine gemeinsame Strategie umsetzen», sagte Stoffel. "Gesetze und Verbote nützen nichts.»

Wie eHealth Ärzten nützen kann, erklärte Stoffel am Beispiel von NewIndex. Die Firma wurde 2000 im Hinblick auf die künftigen tarifarischen Anforderungen unter TARMED gegründet und ist zu 100 Prozent im Besitz der kantonalen Ärztegesellschaften, der FMH und der Ärztekasse. NewIndex erstellt Studien und Auswertungen der ärzteeigenen Daten im Auftrag der Ärzte bei gesundheitsökonomischen Fragen. Ziel von NewIndex ist es, einen gesamtschweizerischen Datenpool zu schaffen. «Mit den ärzteeigenen Daten unterstützt NewIndex die Schweizer Mediziner bei Tarifverhandlungen mit den Versicherern, und ihnen steht damit ein Instrument für allfällige Wirtschaftlichkeitsverfahren zur Verfügung», erklärte Stoffel. Um den Datenpool zu realisieren, wurden zusammen mit der NewIndex gesamtschweizerisch zehn TrustCenter geschaffen, an die 9500 Ärzte Daten liefern. Ein Kodex soll die korrekte Nutzung der Daten gewährleisten. «Die anonymisierten, konsolidierten und aggregierten Daten, welche die NewIndex in der Nationalen Konsolidierungsstelle, der NAKO, auswertet und analysiert, sind seit 2004 unverzichtbar für die Tarifverhandlungen der Kantonalen Ärztegesellschaften und der FMH», sagte Stoffel.

\section{Ungewollte Fremdnutzung der Daten}

Einen anderen Ansatz stellte Mathis Brauchbar vor, Vorstand des Vereins Daten \& Gesundheit. «Die Debatte konzentriert sich stark auf den Datenschutz", sagte Brauchbar. "Doch die Daten nutzen längst andere und wir befinden uns quasi in der digitalen Leibeigenschaft.» Dabei geht es ihm weniger um die Erstnutzung, also dass Supermärkte oder Kreditkartenanbieter Gewinn machen wollen aus unseren $\mathrm{Da}$ ten, sondern um die Zweitnutzung: Anhand unserer Gesundheitsdaten können neue Krankheiten und Epidemien erforscht, Medikamente effizienter entwickelt, Nebenwirkungen nach der Zulassung besser erfasst und einfach Versorgungsforschung durchgeführt werden. «In den Daten steckt viel Wert, aber einer Zweitnutzung stehen viele Hürden im Wege. Selbst wenn wir wollten, könnten wir die Krankheitsdaten gar nicht konsequent verknüpfen, weil die aktuelle Gesetzgebung dies nicht erlaubt.» Profitieren von den Daten würden zurzeit vor allem Firmen wie Amazon, Google, Coop oder Migros, der Einzelne habe nichts davon. Jeder Bürger solle aber Kontrolle bekommen über seine Daten. «Wir könnten ein Teil der Datenökonomie werden.» Die US-Amerikaner seien schon viel weiter: So gibt es erfolgreiche Projekte wie die "Blue Button»-Initiative, die verlangen, dass einem die Daten in geeigneter Form übertragen werden, und im Patientennetzwerk «PatientsLikeMe» berichten und teilen Patienten ihre Erfahrung über ihre Krankheiten und generieren Daten für bessere Therapien. «Das sind Hinweise, dass Ärzte und Firmen ihre

\section{Minuten nach der Operation posten}

Patienten Fotos auf Facebook, das Selfie im Spital gehört für Jugendliche längst dazu.

Dominanz aufgeben müssen und Gesundheitsdaten nicht mehr nur bei ihnen bleiben.» Auch er sei überzeugt, dass Patienten ihre Daten sicherlich nicht so sehr «abschotten» wollten, wie man immer meine. Brauchbar ist sicher, dass wir am Anfang eines Umbruchs sind, ähnlich wie zu Beginn der ersten Computer. "Wir befinden uns im Stadium eines Commodore 64." Ausserdem gäbe es vielleicht eine neue Ethik des Teilens: "Man teilt, weil man denkt, etwas zurückzubekommen.» Damit das Teilen nicht einseitig bleibt, gründete Brauchbar gemeinsam mit anderen Fachleuten 2012 den Verein «Daten und Gesundheit», dessen Ziel genossenschaftlich organisierte Banken für persönliche Gesundheitsdaten sind. Die Bürger entscheiden, welche Daten sie mit wem, zu welchem Zweck und wann teilen wollen, und die Mitglieder legen fest, wie die Gewinne aus der Zweitnutzung von Daten - also durch Verkauf an Dritte investiert werden. Der Verein fordert: Bürger sollten ein Recht auf eine Kopie ihrer Daten haben und sie in geeigneter Form zugestellt bekommen. "Ich bin gespannt, wie schnell wir aus der digitalen Leibeigenschaft herauskommen.»

«Beim Thema Transparenz von Patientendaten müssen wir uns aus der Zwangsjacke «Es muss alles partout geschützt werden> befreien und neue, kreative Wege gehen - dazu gehört auch ein liberalerer Umgang mit Patientendaten», forderte Jacques de Haller. «Nur so können wir uns an die moderne Welt anpassen.» 